\title{
CRISPR deletion of the C9ORF72 promoter in ALS/FTD patient motor neurons abolishes production of dipeptide repeat proteins and rescues neurodegeneration
}

\author{
Gopinath Krishnan ${ }^{1} \cdot$ Yu Zhang ${ }^{1,2} \cdot$ Yuanzheng Gu$^{3} \cdot$ Mark W. Kankel $^{3} \cdot$ Fen-Biao Gao ${ }^{1}$ S Sandra Almeida ${ }^{1}$
}

Received: 30 January 2020 / Revised: 27 March 2020 / Accepted: 27 March 2020 / Published online: 7 April 2020

(c) The Author(s) 2020

GGGGCC $\left(\mathrm{G}_{4} \mathrm{C}_{2}\right)$ repeat expansion in the first intron of C9ORF72 is the most common genetic cause of amyotrophic lateral sclerosis (ALS) and frontotemporal dementia (FTD) $[6,11]$. Brain tissues from affected individuals show characteristic nuclear RNA foci containing the expanded repeat RNAs, as well as neuronal inclusions containing dipeptide repeat (DPR) proteins $[\operatorname{poly}(\mathrm{GA})$, poly(GP), poly(GR), poly(PR), and poly(PA)] resulting from the translation of both sense and antisense repeat RNAs in all reading frames $[4,9,14]$. Although reduced C9ORF72 protein function may contribute to disease [10], the more likely drivers of disease are mechanisms related to a gain of toxic function [7]. Currently, intense efforts are being made to identify disease mechanisms amenable for the development of therapeutic strategies. One promising avenue would be to prevent the production of the expanded repeat RNAs, such as by antisense oligonucleotides [5]. Here, we tested another potential therapeutic approach: CRISPR/Cas9-based targeting of the promoter region.

Gopinath Krishnan and Yu Zhang contributed equally to this work.

Electronic supplementary material The online version of this article (https://doi.org/10.1007/s00401-020-02154-6) contains supplementary material, which is available to authorized users.

Fen-Biao Gao

fen-biao.gao@umassmed.edu

Sandra Almeida

sandra.almeida@umassmed.edu

1 Department of Neurology, University of Massachusetts Medical School, Worcester, MA 01605, USA

2 Department of Neurology, Xinhua Hospital Affiliated to Shanghai Jiao Tong University School of Medicine, Shanghai, China

3 Neuromuscular and Movement Disorders, Biogen, Cambridge, MA 02142, USA
In ALS and FTD patients, transcription initiated at exon1a of C9ORF72 generates RNA species containing $\mathrm{G}_{4} \mathrm{C}_{2}$ repeat expansions that are in turn translated into three DPR proteins, poly(GA), poly(GP), and poly(GR). Thus, we first used reporter constructs to identify the promoter sequence $5^{\prime}$ to exon-1a that is responsible for transcription initiation of expanded repeat RNA. We cloned the 435 nucleotides (nt) upstream of the predicted transcription initiation site, as well as several truncated fragments of the 435-nt sequence, into a luciferase reporter vector (Fig. 1a) and expressed all the constructs in HEK293 cells. Deletion of the first $301 \mathrm{nt}$ did not reduce the relative expression of the reporter gene. However, deleting the remaining $134 \mathrm{nt}$ abolished luciferase expression (Fig. 1a), indicating that the core promoter elements are located in this region. To further characterize this sequence, we also tested a construct lacking the first 394 nt and one lacking the remaining $41 \mathrm{nt}$. Each of these deletions reduced the relative luciferase expression, indicating that both constructs lacked a portion of sequence required for reporter gene expression (Fig. 1a). These experiments indicate that the last $134 \mathrm{nt}$ sequence contains the minimal promoter necessary to drive expression of $\mathrm{G}_{4} \mathrm{C}_{2}$ repeat-containing C9ORF72 transcripts.

To investigate how this deletion affects the production of RNAs containing $\mathrm{G}_{4} \mathrm{C}_{2}$ repeat expansions in C9ORF72 human neurons, we used CRISPR-Cas9 technology to generate a similar deletion $5^{\prime}$ to exon-1a of C9ORF72 in an induced pluripotent stem cell (iPSC) line containing 1000 copies of the $\mathrm{G}_{4} \mathrm{C}_{2}$ repeats [2] (Figs. 1b, c; S1). We selected two iPSC lines containing the promoter deletion and differentiated them and the parental iPSC line into ChAT-positive motor neurons (see Suppl. Information). As expected, the promoter deletion eliminated the expression of C9ORF72Variant 3 (V3), whose transcription starts on exon-1a (Fig. 1b, d). In contrast, the RNA level of C9ORF72-V2, whose translation starts on exon $1 \mathrm{~b}$, or the expression of C9ORF72-antisense RNA, was not significantly affected 
Fig. 1 Deletion of the C9ORF72 promoter region. a Schematic of the different promoter deletions analyzed in the luciferase reporter assay and quantification of the relative expression of firefly luciferase (FLuc)/renilla luciferase (RLuc) in HEK293 cells $(n=3$ independent transfections). b Schematic of the C9ORF72 locus in parental and promoter deletion iPSC-derived neurons. Arrows indicate the site of transcription initiation for the different variants. Only variants containing exon 1a are expected to be affected by the deletion; the variant containing exon $1 \mathrm{~b}$ is predicted to use a different promoter. c CRISPR-edited iPSC lines are homozygous for the 140-bp deletion as indicated by the PCR analysis. $\mathbf{d - f}$ Four-week-old motor neurons from parental and promoter deletion lines were analyzed for expression of C9ORF72-V3, C9ORF72-V2, and C9ORF72antisense RNAs $(n=3$ independent differentiations). g C9ORF72 protein levels in 4-week-old parental and promoter deletion motor neurons $(n=3$ independent differentiations). Values are mean \pm SEM. $* p<0.05, * * p<0.01$, $* * * p<0.001$ (a, e-g, one-way ANOVA; d, Welch's $t$ test). $n s$ not significant a
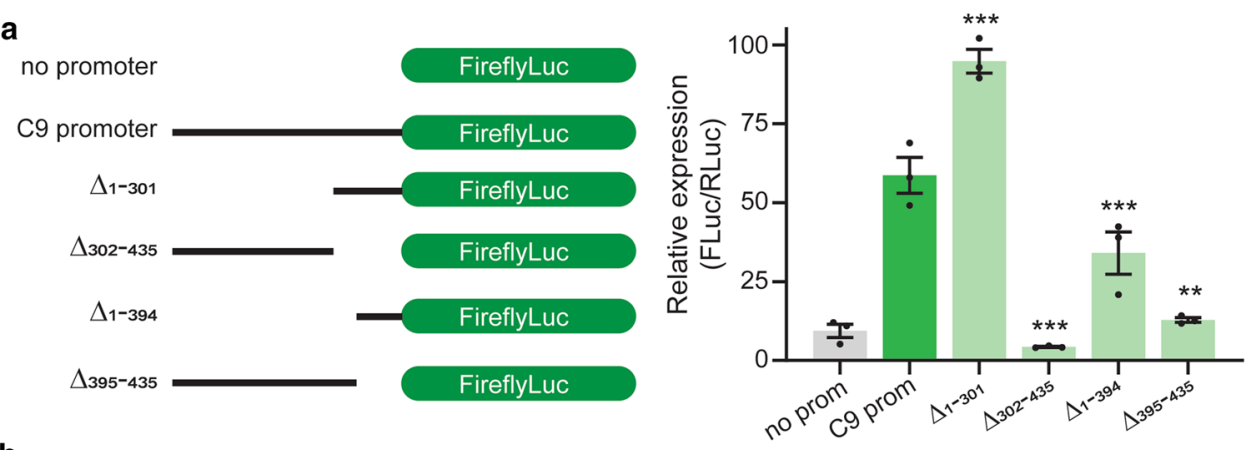

b

C9 - Parental:

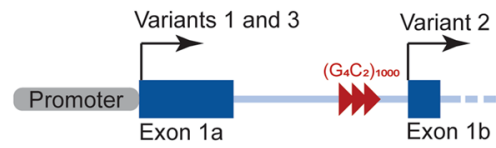

C9 - Promoter deletion:

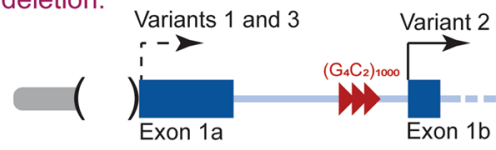

d

C9ORF72-V3

e

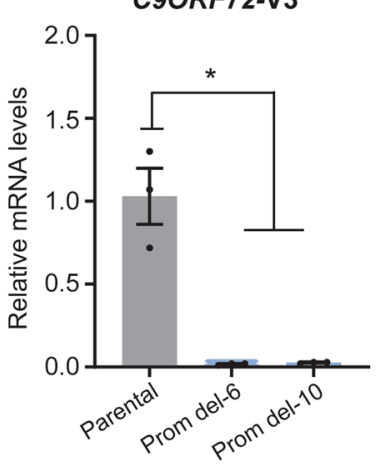

C9ORF72-V2

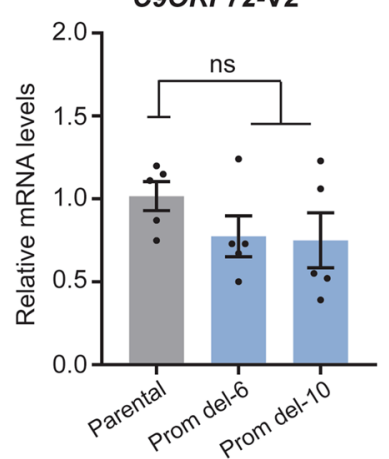

C

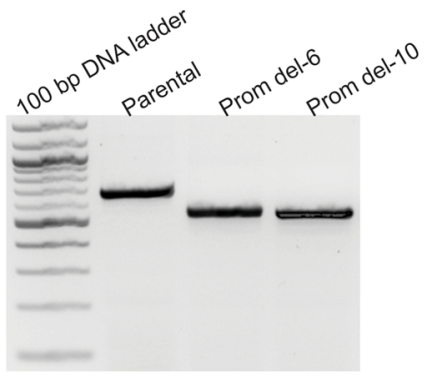

f

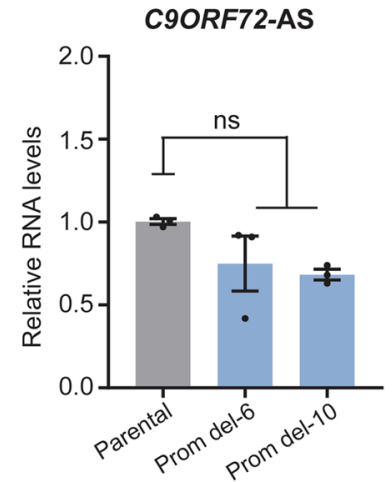

g

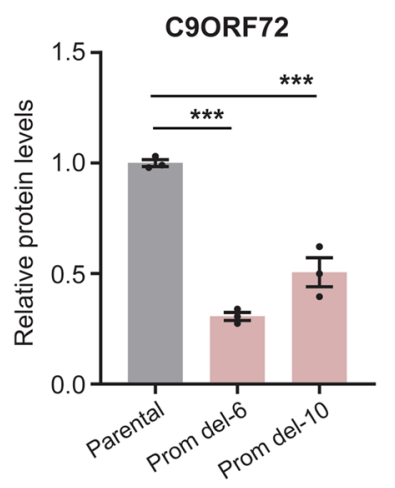

(Fig. 1e-f), consistent with the fact that these transcripts have their own promoters [6]. In iPSCs and motor neurons harboring the promoter deletion, we detected a reduction in the levels of full-length C9ORF72 protein (Figs. 1g; S2), likely due to loss of C9ORF72-V3 expression.

Since RNAs containing the $\mathrm{G}_{4} \mathrm{C}_{2}$ repeat expansion lead to the production of DPR proteins, we next measured DPR protein levels in parental and promoter deletion motor neuron cultures using Meso Scale Discovery immunoassays. We found that expression of poly(GA), poly(GP), and poly(GR) was almost completely abolished in the motor neuron cultures harboring the promoter deletion (Fig. $2 \mathrm{a}-\mathrm{c}$ ). We then examined the functional consequence of reducing DPR levels in these neurons, using axonal degeneration as our 
a

Poly(GA)

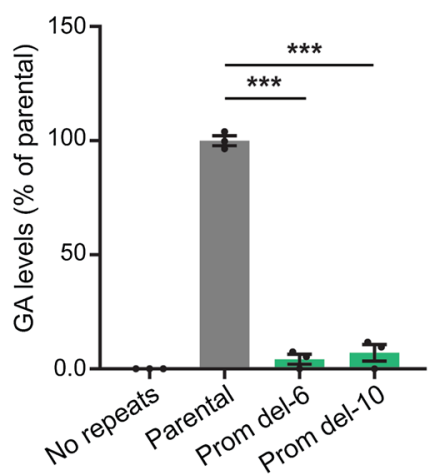

d

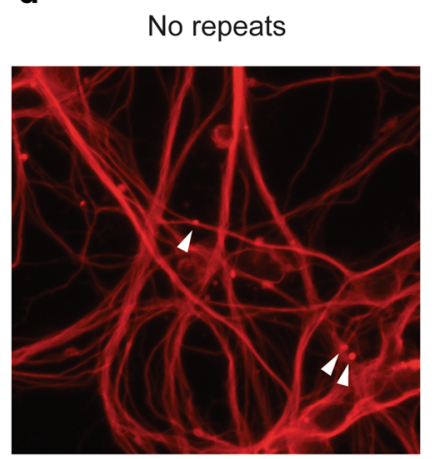

Promoter del-6

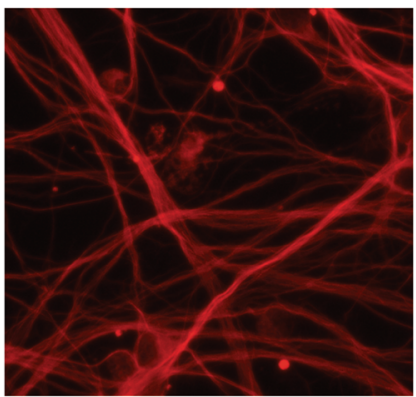

b

$\operatorname{Poly}(G P)$
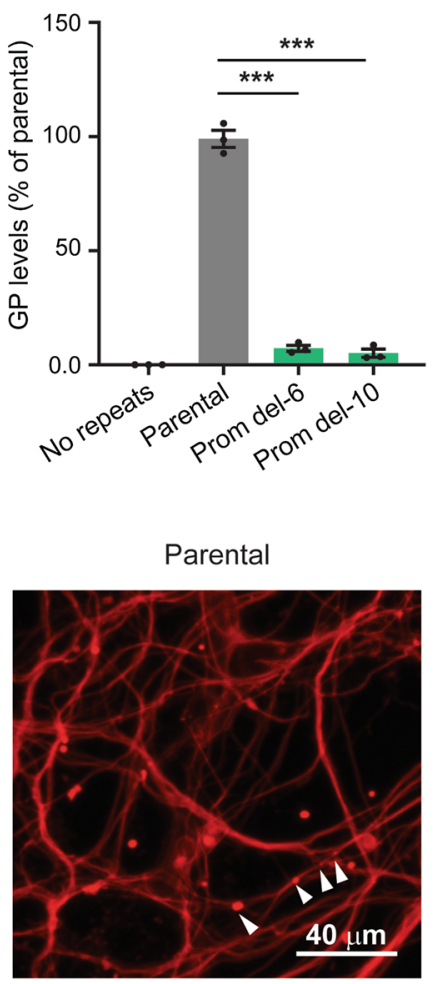

Promoter del-10

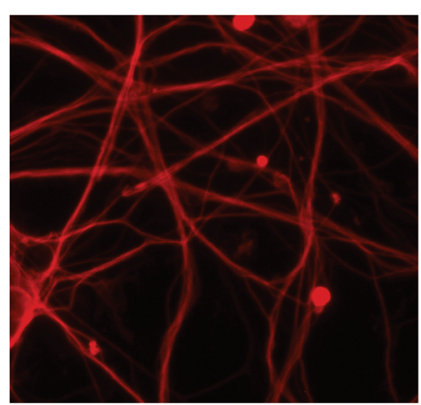

C

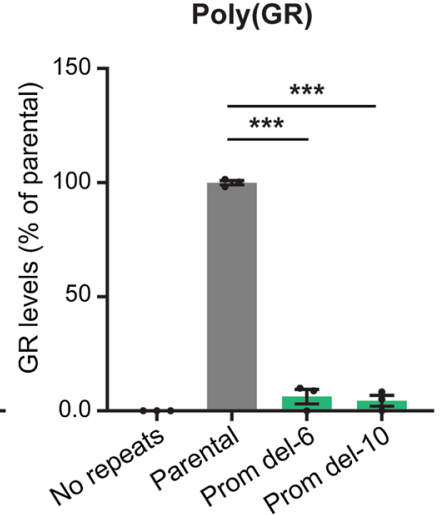

e

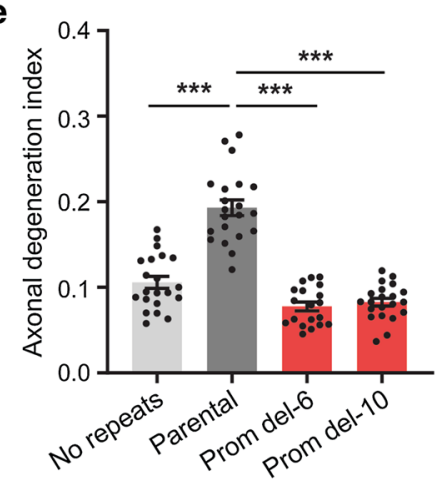

f

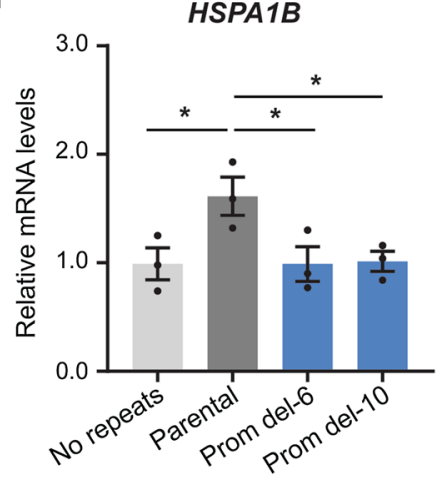

Fig. 2 Effects of C9ORF72 promoter deletion in iPSC-derived neurons. a-c Poly(GA), poly(GP), and poly(GR) levels in 1.5-month-old parental neurons, neurons without repeats, and promoter deletion motor neurons ( $n=3$ independent differentiations) were measured with Meso Scale Discovery immunoassays. The poly(GA) assay was done in a blinded manner at Biogen. d Representative immunofluorescence images of the axonal degeneration assay done with the marker $\beta \mathrm{III}$-tubulin $\left(\mathrm{TUJ}^{+}\right)$, which revealed swollen varicosities and axonal fragments. Arrowheads indicate fragmented axons. e Axonal

scoring metric as we reported recently [12]. Deletion of the promoter region prevented the increase in axonal degeneration of parental motor neurons upon withdrawal of neurotrophic factors for 2 weeks (Figs. 2d, e; S3), a phenotype we previously described in neurons differentiated from two pairs of isogenic C9ORF72 iPSC lines [12]. The promoter degeneration assessed by measuring the ratio of fragmented axons over the total $\mathrm{TUJ}_{1}{ }^{+}$area 2 weeks after withdrawal of neurotrophic factors. Six to eight randomly selected fields were analyzed for each condition and each neuronal culture. Each independent data point represents one field, ( $n=3$ independent differentiations). f Relative expression of HSPA1B in 10-week-old motor neuron cultures $(n=3$ independent differentiations). Values are mean \pm SEM. $* p<0.05$, $* * * p<0.001$ (one-way ANOVA, Dunnett's multiple comparisons test)

deletion also prevented upregulation of the expression of the heat shock protein $H S P A 1 B$ mRNA (Fig. 2f), a phenotype detected in ALS/FTD patient brain tissues and C9ORF72 iPSC-derived motor neurons $[3,8]$.

Our results indicate that deletion of the $134 \mathrm{nt}$ minimal promoter $5^{\prime}$ to exon-1a in C9ORF72 prevents both 
the production of sense RNAs containing expanded $\mathrm{G}_{4} \mathrm{C}_{2}$ repeats and the activation of downstream neurodegeneration pathways. These findings also suggest that sense repeat RNA and its translation products are responsible for the observed neurodegenerative phenotypes, at least in this experimental system. The promoter deletion also partially decreased C9ORF72 protein levels. However, in the absence of DPR proteins, loss of C9ORF72 does not cause neurodegeneration in mice or iPSC-derived motor neurons [1, 10]. Improved versions of the CRISPR interference are now being tested in vivo to silence gene transcription without cutting genomic DNA [13]. Thus, our proof-of-concept study suggests that CRISPR/Cas9-based targeting of the promoter region to eliminate sense repeat RNA and its toxic translation products may be a potentially useful therapeutic approach for C9ORF72-ALS/FTD, especially before significant accumulation of DPR proteins.

Acknowledgements This work was supported by grants from the NIH (R37NS057553, R01NS101986, and R01NS093097 to F.B.G. and R21NS112766 to S.A.); the MDA Foundation and the Target ALS Foundation (F.-B.G.); the Frick Foundation for ALS Research, the Alzheimer's Association (2016-NIRG-396129), and the ALS Association (S.A.); and the National Key R\&D Program of China (2017YFC1310300) (Y.Z.)

Open Access This article is licensed under a Creative Commons Attribution 4.0 International License, which permits use, sharing, adaptation, distribution and reproduction in any medium or format, as long as you give appropriate credit to the original author(s) and the source, provide a link to the Creative Commons licence, and indicate if changes were made. The images or other third party material in this article are included in the article's Creative Commons licence, unless indicated otherwise in a credit line to the material. If material is not included in the article's Creative Commons licence and your intended use is not permitted by statutory regulation or exceeds the permitted use, you will need to obtain permission directly from the copyright holder. To view a copy of this licence, visit http://creativecommons.org/licenses/by/4.0/.

\section{References}

1. Abo-Rady M, Kalmbach N, Pal A, Schludi C, Janosch A, Richter $\mathrm{T}$ et al (2020) Knocking out C9ORF72 exacerbates axonal trafficking defects associated with hexanucleotide repeat expansion and reduces levels of heat shock proteins. Stem Cell Rep 14:390-405

2. Almeida S, Gascon E, Tran H, Chou HJ, Gendron TF, Degroot S et al (2013) Modeling key pathological features of frontotemporal dementia with C9ORF72 repeat expansion in iPSC-derived human neurons. Acta Neuropathol 126:385-399

3. Almeida S, Krishnan G, Rushe M, Gu Y, Kankel MW, Gao FB (2019) Production of poly(GA) in C9ORF72 patient motor neurons derived from induced pluripotent stem cells. Acta Neuropathol 138:1099-1101

4. Ash PE, Bieniek KF, Gendron TF, Caulfield T, Lin WL, Dejesus-Hernandez $\mathrm{M}$ et al (2013) Unconventional translation of C9ORF72 GGGGCC expansion generates insoluble polypeptides specific to c9FTD/ALS. Neuron 77:639-646

5. Bennett CF, Krainer AR, Cleveland DW (2019) Antisense oligonucleotide therapies for neurodegenerative diseases. Annu Rev Neurosci 42:385-406

6. De Jesus-Hernandez M, Mackenzie IR, Boeve BF, Boxer AL, Baker M, Rutherford NJ et al (2011) Expanded GGGGCC hexanucleotide repeat in noncoding region of C9ORF72 causes chromosome 9p-linked FTD and ALS. Neuron 72:245-256

7. Gao FB, Almeida S, Lopez-Gonzalez R (2017) Dysregulated molecular pathways in amyotrophic lateral sclerosis-frontotemporal dementia spectrum disorder. EMBO J 36:2931-2950

8. Mordes DA, Prudencio M, Goodman LD, Klim JR, Moccia R et al (2018) Dipeptide repeat proteins activate a heat shock response found in C9ORF72-ALS/FTLD patients. Acta Neuropathol Commun 6:55

9. Mori K, Weng SM, Arzberger T, May S, Rentzsch K, Kremmer E et al (2013) The C9orf72 GGGGCC repeat is translated into aggregating dipeptide-repeat proteins in FTLD/ALS. Science 339:1335-1338

10. O'Rourke JG, Bogdanik L, Yáñez A, Lall D, Wolf AJ, Muhammad AK et al (2016) C9orf72 is required for proper macrophage and microglial function in mice. Science 351:1324-1329

11. Renton AE, Majounie E, Waite A, Simon-Sanchez J, Rollinson S, Gibbs JR et al (2011) A hexanucleotide repeat expansion in C9ORF72 is the cause of chromosome 9p21-linked ALS-FTD. Neuron 72:257-268

12. Yuva-Aydemir Y, Almeida S, Krishnan G, Gendron TF, Gao FB (2019) Transcription elongation factor AFF2/FMR2 regulates expression of expanded GGGGCC repeat-containing C9ORF72 allele in ALS/FTD. Nat Commun 10:5466

13. Zheng Y, Shen W, Zhang J, Yang B, Liu YN et al (2018) CRISPR interference-based specific and efficient gene inactivation in the brain. Nat Neurosci 21:447-454

14. Zu T, Liu Y, Bañez-Coronel M, Reid T, Pletnikova O, Lewis J et al (2013) RAN proteins and RNA foci from antisense transcripts in C9ORF72 ALS and frontotemporal dementia. Proc Natl Acad Sci USA 110:E4968-E4977

Publisher's Note Springer Nature remains neutral with regard to jurisdictional claims in published maps and institutional affiliations. 